Pacific Journal of Mathematic 


\title{
DESARGUES' LAW AND THE REPRESENTATION OF PRIMARY LATTICES
}

\author{
G. S. MoNK
}

In an earlier work of $B$. Jónsson and the author it was shown that an Arguesian primary lattice of geometric dimension at least 3 can be represented as the submodule lattice of a finitely generated module over a completely primary uniserial ring. Inasmuch as the class of primary lattices includes the class of subspace lattices of (nondegenerate) projective geometries, two questions then naturally arise: (1) Is a primary lattice of geometric dimension at least 4 Arguesian? (2) Is an Arguesian primary lattice of geometric dimension 2 representable?

The first question is answered in the affirmative in $\$ 1$, thus showing that the abovementioned paper subsumes the results of $\mathrm{E}$. Inaba on the representation of primary lattices of geometric dimension at least 4 . A counter example is given in $\$ 2$ showing that an Arguesian lattice of geometric dimension 2 cannot, in general, be represented, but for reasons far deeper than the cardinality arguments given for the representability or nonrepresentability of subspace lattices of 1-dimensional projective geometries.

We will assume that the reader is familiar with the first six sections of [3] (henceforth referred to as PAL) and will adopt the notation and terminology of that work.

It is well known that the class of finite dimensional simple complemented modular lattices of dimension 3 or more coincides with the class of subspace lattices of (nondegenerate) projective geometries of dimension at least 2, and that those lattices which are Arguesian correspond to Arguesian geometries, i.e., those geometries that can be coordinatized by division rings. We will freely use these facts to translate arguments in geometry to arguments in lattice theory. In particular, they will be applied to complemented intervals in primary lattices, which, in view of 6.3 of PAL, are simple.

1. Lemma 1.1. Given cycles $\left\{w_{i}\right\}_{i=0}^{n}$ in a primary lattice $L$ such that $w_{i} \not \leq \sum\left\{w_{j} \mid j \neq i\right\}$, there are cycles $\left\{x_{i}\right\}_{i=0}^{n}$ of $L$ and a permutation $\varphi$ of $\{0,1,2, \cdots, n\}$ such that $\sum_{0}^{j} w_{\varphi(i)}=\dot{\sum}_{0}^{j} x_{i}$ for $0 \leqq j \leqq n$, and $0<d\left[x_{j}\right] \leqq d\left[x_{j-1}\right]$ for $j=1,2, \cdots, n$.

Proof. We will show by induction on $m$ that for $0 \leqq m \leqq n$, there is a set of cycles $\left\{\left\{w_{\imath j}\right\}_{j=\imath}^{n}\right\}_{i=0}^{m}$ such that:

(1) $\left\{w_{0 i}\right\}_{i=0}^{n}=\left\{w_{j}\right\}_{j=0}^{n}$, 
$(2)_{m} d\left[w_{m, m}\right] \geqq d\left[w_{m, j}\right]$, $j \geqq m$,

( 3$)_{m} 0<d\left[w_{i i}\right] \leqq d\left[w_{i-1, i-1}\right]$, $1 \leqq i \leqq m$,

$(4)_{m} \quad \sum_{0}^{m-1} w_{i i}+w_{k j}=\sum_{0}^{m-1} w_{\imath i}+w_{m j}$, $k<m \leqq j$,

(5) $)_{m} \quad \sum_{0}^{j} w_{0 i}=\dot{\sum}_{0}^{j} w_{i i}$,

$j \leqq m$.

For then, taking $m=n$, we will have the desired set of cycles by letting $x_{i}$ be $w_{i i}$ and $\varphi$ the permutation given by $w_{0 i}=w_{\varphi(i)}$. Inasmuch as such a set of cycles is given for $m=0$ by taking $w_{00}$ to be the element of $\left\{w_{i}\right\}_{i=0}^{n}$ of greatest dimension and $\left\{w_{0 i}\right\}_{i=1}^{n}$ the remaining elements of $\left\{w_{i}\right\}_{2=0}^{n}$, we can assume that we have such a set for $0<m<n$. Then, setting $d\left[w_{m m}\right]=r$, we infer from 4.14 of PAL that, for $j>m, \sum_{0}^{m} w_{i i}[r]$ is geometric in $\left[0, \sum_{0}^{m} w_{i i}[r]+w_{m j}\right]$, so that, by 5.2 of PAL, it has a complement $w_{m+1, j}$ in this interval. It then follows that

$$
\sum_{0}^{m} w_{i i}+w_{m+1 . j}=\sum_{0}^{m} w_{i i}+w_{m j},
$$

whence, by a routine argument, we infer that

$$
\left[0, w_{m+1, j}\right] \cong\left[\left(\sum_{0}^{m} w_{i i}\right) w_{m j}, w_{m, j}\right] .
$$

This, together with the assumption that $w_{j} \npreceq \sum\left\{w_{i} \mid i \neq j\right\}$ yields that $w_{m+1, j}$ is a cycle such that

$$
0<d\left[w_{m+1, j}\right] \leqq d\left[w_{m, j}\right] .
$$

Now, if $s$ has the property that $w_{m+1, s}$ is of greatest dimension among the elements $\left\{w_{m+1, j}\right\}_{j=m+1}^{n}$, set

$$
w_{i j}^{\prime}= \begin{cases}w_{i j} & j \neq s, m+1 \\ w_{i s} & j=m+1 \\ w_{i, m+1} & j=s .\end{cases}
$$

Observe that since $s$ and $m+1$ are both greater than $m$, the cycles $\left\{\left\{w_{2 j}^{\prime}\right\}_{j=i}^{n}\right\}_{i=0}^{m}$ satisfy the formulas $(1)_{m}$ through $(5)_{m}$, and that $\left.\left\{w_{i j}^{\prime}\right\}_{j=i}^{n}\right\}_{i=0}^{m+1}$ satisfy (6) and (7) as well. It is clear that (1) $)_{m+1}$ and (2) $)_{m+1}$ hold, while from (7), (2) $m$ and $(3)_{m}$ we conclude that $(3)_{m+1}$ holds. That $(4)_{m+1}$ holds in the case $k=m$ follows from (6). On the other hand, for $k<m$, we infer from $(4)_{m}$ and (6) that

$$
w_{m m}^{\prime}+\sum_{0}^{m-1} w_{i i}^{\prime}+w_{k j}^{\prime}=w_{m m}^{\prime}+\sum_{0}^{m-1} w_{i i}^{\prime}+w_{m j}^{\prime}=\sum_{0}^{m} w_{i i}^{\prime}+w_{m+1, j}^{\prime},
$$

so that $(4)_{m+1}$ holds. Finally, we infer from $(5)_{m}$ and $(4)_{m+1}$ that $\sum_{0}^{m+1} w_{0 i}=\sum_{0}^{m+1} w_{i i}$, which together with $(5)_{m}$ and the fact that $\left(\sum^{m} w_{i i}\right) w_{m+1, m+1}=0$ yields $(5)_{m+1}$ and completes the proof of the lemma.

Notation. Given a primary lattice $L$, denote the sum of all atoms 
of $L$ by $p(L)$.

For any element $x$ of a primary lattice $L$, we clearly have that $x[1]=x p(L)$.

As an immediate consequence of 4.14 of PAL and 1.1, we have

COROLLARY 1.2. Given cycles $\left\{w_{i}\right\}_{i=0}^{n}$ in a primary lattice $L$ such that $w_{i} \not \sum\left\{w_{j} \mid j \neq i\right\}$, the element $\left(\sum_{0}^{n} w_{i}\right)[1]$ of $[0, p(L)]$ is of dimension $n+1$.

LEMma 1.3. Given cycles $u, w_{0}, w_{1}, w_{2}$ and $w_{3}$ in a primary lattice $L$ such that $u$ is contained in $\sum_{0}^{3} w_{i}$ and is disjoint from $\sum\left\{w_{i} \mid i \neq j\right\}$ for $j=0,1,2,3$, there is an integer $n=0,1,2,3$ and a cycle $s$ of $L$ disjoint from $\sum\left\{w_{i} \mid i \neq j\right\}$ for $j=0,1,2,3$ such that $w_{n} \leqq s+\sum\left\{w_{i} \mid i \neq n\right\}$.

Proof. Letting $W_{i}=\sum\left\{w_{j} \mid j \neq i\right\}$, we might as well assume that $w_{i} \not \equiv W_{i}$ for every $i$, since otherwise $u$ would suffice. We then infer from 1.1 that there are cycles $\left\{x_{i}\right\}_{i=0}^{3}$ and a permutation $\varphi$ of $\{0,1,2,3\}$ such that $\sum_{0}^{j} w_{\varphi(i)}=\dot{\sum}_{0}^{j} x_{i}$ and $0<d\left[x_{j}\right] \leqq d\left[x_{j-1}\right]$ for $1 \leqq j \leqq 3$. Thus, setting $k=d\left[x_{3}\right]$ and

$$
a=\left(\dot{\sum}_{0}^{3} x_{i}\right)[k]=\dot{\sum}_{0}^{3} x_{i}[k],
$$

we have that $[0, a]$ is regular and

$$
a \geqq\left(\sum_{0}^{3} x_{i}\right)[1] \geqq\left(\sum_{0}^{3} w_{\varphi(i)}\right)[1] \geqq u
$$

so that, by 5.3 of PAL, $u$ is contained in a point $s$ of $[0, a]$. We infer immediately from 4.14 of PAL that $s$ is disjoint from $W_{i}$ for every $i$, since $u$ has this property. Moreover,

$$
0=s W_{\varphi(3)}=s\left(x_{0}+x_{1}+x_{2}\right)=s\left(x_{0}[k]+x_{1}[k]+x_{2}[k]\right),
$$

whence, by a dimension argument,

$$
a=s+x_{0}[k]+x_{1}[k]+x_{2}[k],
$$

and

$$
s+W_{\varphi(3)}=s+x_{0}+x_{1}+x_{2} \geqq a \geqq x_{3}[k]=x_{3} .
$$

Inasmuch as $s+W_{\varphi(3)}$ also contains $x_{0}+x_{1}+x_{2}$, we conclude that $s+W_{\varphi(3)} \geqq w_{\varphi(3)}$ and $s$ is the desired element.

Lemma 1.4. Let $x_{0}, x_{1}, x_{2}$ be elements of a primary lattice $L$ such that $x_{1}$ and $x_{2}$ are cycles and $\left(x_{0}+x_{1}\right)[1] \neq\left(x_{0}+x_{2}\right)[1]$. Then $\left(x_{0}+x_{1}\right)\left(x_{0}+x_{2}\right)=x_{0}$. 
Proof. Observe that $x_{0}+x_{1}$ and $x_{0}+x_{2}$ are cycles in the primary lattice $L^{\prime}=\left[x_{0}, 1\right]$ such that

$$
\left(x_{0}+x_{1}\right) p\left(L^{\prime}\right) \neq\left(x_{0}+x_{2}\right) p\left(L^{\prime}\right) .
$$

Thus, by 4.14 of PAL, $\left(x_{0}+x_{1}\right)\left(x_{0}+x_{2}\right)=x_{0}$, the zero element of $L^{\prime}$.

(1.5) Theorem. Given cycles $a_{0}, a_{1}, a_{2}, b_{0}, b_{1}, b_{2}, c$, in a primary lattice $L$, let $a_{3}=c=b_{3}$, and

$$
\begin{aligned}
& A_{i}=\sum\left\{a_{j} \mid j \neq i\right\}, B_{i}=\sum\left\{b_{j} \mid j \neq i\right\}, \quad i=0,1,2,3, \\
& p_{j}=\left(a_{i}+a_{k}\right)\left(b_{i}+b_{k}\right), \quad i \neq j \neq k, i, j, k,=\{0,1,2\} .
\end{aligned}
$$

If $a_{i}+c=a_{i}+b_{i}=b_{i}+c$ for $i=0,1,2$, and there are cycles $\left\{t_{i}\right\}_{i=0}^{3}$ in $L$ such that $d\left[t_{i}\right] \geqq d[c]$ and $t_{i} A_{i}=0$ for $i=0,1,2,3$, then $a_{0} \leqq a_{1}+p_{2}\left(p_{0}+p_{1}\right)$.

Proof. Case 1. $\left\{a_{0}, a_{1}, a_{2}\right\} \perp$ or $A_{0}[1] \geqq A_{1}[1], A_{2}[1]$. We will first show that there is an atom $u$ of $L$ disjoint from $A_{i}(1)$ for $i=0,1,2,3$. There is clearly such an element under any of the conditions:

$$
A_{0}[1] \geqq A_{1}[1], A_{2}[1] ;\left\{a_{0}, a_{1}, a_{2}, a_{3}\right\} ;
$$

that

$a_{i} \leqq A_{i}$ for some $i=0,1,2,3$. Thus we might as well assume

$$
\left\{a_{0}, a_{1}, a_{2}\right\} \perp ; a_{3} A_{3}>0 ; a_{i} \not A_{i} \quad i=0,1,2,3 .
$$

First observe that according to 1.2 , the elements $\left\{A_{i}[1]\right\}_{i=0}^{3}$ are planes in the 4-dimensional simple complemented modular lattice [0, $\left.\left(\sum_{3}^{0} a_{i}\right)[1]\right]$. Furthermore, inasmuch as $a_{3}$ is a cycle and

$$
0=\left(a_{0}+a_{1}\right)\left(a_{0}+a_{2}\right)\left(a_{1}+a_{2}\right) \geqq a_{3}\left(a_{0}+a_{1}\right)\left(a_{0}+a_{2}\right)\left(a_{1}+a_{2}\right),
$$

we have that $\left\{a_{3}, a_{i}, a_{j}\right\} \perp$ for some $i$ and $j$, so that for $k$ distinct from $i, j$ and $3, A_{k}[1]=a_{i}[1]+a_{j}[1]+a_{3}[1]$. Since the assumption that $a_{3} A_{3}>0$ implies that $a_{3}[1] \leqq A_{3}[1]$, we then infer that $A_{k}[1] \leqq A_{3}[1]$. But then $A_{k}[1]=A_{3}[1]$, because these elements are both planes in $\left[0,\left(\sum_{0}^{3} a_{i}\right)[1]\right]$. We can therefore assume that the planes $A_{0}[1], A_{1}[1]$ and $A_{2}[1]$ are distinct, for otherwise we would immediately have the existence of the desired atom $u$. But then, if $A_{i}[1] A_{k}[1] \leqq A_{j}[1]$ for $i, j$ and $k$ distinct in $\{0,1,2\}$, we infer from 1.4 that

$$
\begin{aligned}
& A_{i}[1] A_{k}[1] \leqq A_{j}[1] A_{k}[1]=\left(a_{i}+a_{3}\right)[1], \\
& A_{i}[1] A_{k}[1] \leqq A_{i}[1] A_{j}[1]=\left(a_{k}+a_{3}\right)[1]
\end{aligned}
$$

Inasmuch as $\left(a_{i}+a_{3}\right)[1]$ and $\left(a_{k}+a_{3}\right)[1]$ are lines and $A_{i}[1]$ and $A_{k}[1]$ are distinct planes in $\left.\left[0, \sum_{0}^{3} a_{i}\right)[1]\right]$, these formulas are equalities, and the points $a_{0}[1], a_{1}[1]$ and $a_{2}[1]$ are contained on the line $\left(a_{i}+a_{3}\right)[1]$. 
This contradicts, by 4.14 of PAL, the independence of the cycles $a_{0}, a_{1}$ and $a_{2}$, and we conclude that $A_{i}[1] A_{k}[1] \not A_{j}[1]$ for $i, j$ and $k$ distinct in $\{0,1,2\}$. From this we see that the desired point $u$ of $\left[0,\left(\sum_{0}^{3} a_{i}\right)[1]\right]$ exists by considering the dual situation in which there are four points in a 4-dimensional simple complemented modular lattice of which exactly three are distinct and are not collinear. It is clear that in such a case there is a plane not containing any of the points.

Thus we can apply Lemma 1.3 to obtain a cycle $s$ of $L$ disjoint from $A_{i}$ for every $i$ and such that $s+A_{n} \geqq \alpha_{n}$ for some $n$. Then, letting

$$
\begin{array}{ll}
a_{i}^{\prime} & =\left(s+a_{i}\right) A_{n}, \quad b_{\imath}^{\prime}=\left(s+b_{i}\right) A_{n}, \quad i=0,1,2 \\
c^{\prime} & =(s+c) A_{n},
\end{array}
$$

it is easily seen that $d\left[c^{\prime}\right] \leqq d[c]$, and $a_{i}^{\prime}+b_{i}^{\prime}=a_{i}^{\prime}+c^{\prime}=b_{i}^{\prime}+c^{\prime}$ for $i=0,1,2$. Choosing a cycle $c_{1} \leqq t_{n}$ of the same dimension as $c^{\prime}$, and a cycle $c_{2}$ such that $c_{1} \dot{+} c^{\prime}=c_{2} \dot{+} c^{\prime}=c_{1} \dot{+} c_{2}$, let $d_{i}=\left(c_{1}+a_{\imath}^{\prime}\right)\left(c_{2}+b_{i}^{\prime}\right)$ for $i=0,1,2$, and

$$
g_{i}=\left(c_{1}+d_{j}+d_{k}\right)\left(c_{2}+d_{j}+d_{k}\right) A_{n}, \quad i \neq j \neq k \neq i, i=0,1,2,
$$

and cyclically. It can then be shown that

$a_{i}+d_{i}=c_{1}+a_{i}^{\prime}=e_{1}+d_{i}$, and $b_{i}^{\prime}+d_{i}=c_{2}+b_{i}^{\prime}=c_{2}+d_{i}$ for $i=0,1,2$.

Clearly

since

$$
\begin{aligned}
g_{2}\left(g_{0}+g_{1}\right) & \geqq\left(d_{0}+d_{1}\right) A_{n}\left[\left(d_{1}+d_{2}\right) A_{n}+\left(d_{0}+d_{2}\right) A_{n}\right] \\
& =\left(d_{0}+d_{1}\right) A_{n}\left[d_{1}+d_{2}+\left(d_{0}+d_{2}\right) A_{n}\right] \\
& =\left(d_{0}+d_{1}\right) A_{n},
\end{aligned}
$$

Thus

$$
d_{2}+A_{n} \geqq d_{2}+a_{2}^{\prime}+a_{0}^{\prime}=d_{2}+c_{1}+a_{0}^{\prime} \geqq d_{0} \text {. }
$$

$$
a_{1}^{\prime}+g_{2}\left(g_{0}+g_{1}\right) \geqq\left(a_{1}^{\prime}+d_{0}+d_{1}\right) A_{n}=\left(a_{1}^{\prime}+d_{0}+c_{1}\right) A_{n} \geqq a_{0}^{\prime} .
$$

On the other hand, since $s$ is disjoint from every $A_{i}$, we have that for $j, k=0,1,2$

$$
\begin{aligned}
& s\left(a_{j}+a_{k}+b_{j}+b_{k}\right)=s\left(c+a_{j}+a_{k}\right)=0, \\
& s\left(p_{0}+p_{1}+p_{2}\right) \leqq s\left(a_{0}+a_{1}+a_{2}\right)=0,
\end{aligned}
$$

so that $\left\{s, a_{j}+a_{k}, b_{j}+b_{k}\right\} D$, and $\left\{s, p_{2}, p_{0}+p_{1}\right\} D$. Consequently

$$
\begin{aligned}
g_{0} & =\left(c_{1}+a_{1}^{\prime}+a_{2}^{\prime}\right)\left(c_{2}+b_{1}^{\prime}+b_{2}^{\prime}\right) A_{n}=\left(a_{1}^{\prime}+a_{2}^{\prime}\right)\left(b_{1}^{\prime}+b_{2}^{\prime}\right) \\
& =\left(s+a_{1}+a_{2}\right)\left(s+b_{1}+b_{2}\right) A_{n}=\left(s+p_{0}\right) A_{n}
\end{aligned}
$$

and cyclically, whence

$$
\begin{aligned}
g_{2}\left(g_{0}+g_{1}\right) & =\left(s+p_{2}\right) A_{n}\left[\left(s+p_{0}\right) A_{n}+\left(s+p_{1}\right) A_{n}\right] \\
& =\left[s+p_{2}\left(p_{0}+p_{1}\right)\right] A_{n},
\end{aligned}
$$


and

$$
a_{1}^{\prime}+g_{2}\left(g_{0}+g_{1}\right)=\left[a_{1}^{\prime}+s+p_{2}\left(p_{0}+p_{1}\right)\right] A n .
$$

Combining (8) and (9), we have

$$
a_{0}^{\prime} \leqq a_{1}^{\prime}+s+p_{2}\left(p_{0}+p_{1}\right)
$$

and

$$
s+a_{0} \leqq s+a_{1}+p_{2}\left(p_{0}+p_{1}\right) \text {. }
$$

Multiplying both sides of this formula by $a_{0}+a_{1}$ and observing that $s\left(a_{0}+a_{1}\right) \leqq s A_{3}=0$, we conclude that $a_{0} \leqq a_{1}+p_{2}\left(p_{0}+p_{1}\right)$.

Case 2. $\left\{a_{0}, a_{1}, a_{2}\right\}$ 土 and $A_{i}[1] \leqq A_{0}[1]$ for $i=1$ or 2 . Let $u=$ $\left(a_{0}+a_{1}\right)\left(a_{0}+a_{2}\right)\left(a_{1}+a_{2}\right)$, and $a_{i}^{\prime}=a_{i}+u$ for $i=0,1,2,3$. Then $a_{i}^{\prime}+a_{j}^{\prime}=$ $a_{i}+a_{j}$ for $i, j=0,1,2,\left(a_{0}^{\prime}+a_{1}^{\prime}\right)\left(a_{0}^{\prime}+a_{2}^{\prime}\right)\left(a_{1}^{\prime}+a_{2}^{\prime}\right)=u$, and $\left\{a_{0}^{\prime}, a_{1}^{\prime}, a_{2}^{\prime}\right\} \perp$ in [ $u, 1]$. Thus, letting $t_{i}^{\prime}=t_{i}+u$ for $i=0,1,2,3$, and $A_{0}^{\prime}=a_{1}^{\prime}+a_{2}^{\prime}+a_{3}^{\prime}$ and cyclically, since

$$
\begin{gathered}
t_{i}^{\prime} A_{i}^{\prime}=\left(t_{i}+u\right) A_{i}=u+t_{i} A_{i}=u, \\
d\left[t_{i}^{\prime}\right]=d\left[t_{i}\right]+d[u]-d\left[t_{i} u\right]=d\left[t_{i}\right]+d[u] \geqq d\left[c^{\prime}\right],
\end{gathered}
$$

we can apply Case 1 to $\left\{a_{i}^{\prime}, b_{i}^{\prime}\right\}_{i=0}^{3}$ to conclude that

$$
a_{0}^{\prime} \leqq a_{1}^{\prime}+p_{2}^{\prime}\left(p_{0}^{\prime}+p_{1}^{\prime}\right) \text {, }
$$

where

$$
p_{i}^{\prime}=\left(a_{j}^{\prime}+a_{k}^{\prime}\right)\left(b_{j}^{\prime}+b_{k}^{\prime}\right)=\left(a_{j}+a_{k}\right)\left(b_{j}+b_{k}+u\right)=u+p_{i} .
$$

We infer from (10) that

$$
\begin{aligned}
a_{0} & \leqq u+a_{1}+\left(u+p_{2}\right)\left(u+p_{0}+p_{1}\right) \\
& =u+a_{1}+p_{2} B_{3}\left(u+p_{0}+p_{1}\right) \\
& =u+a_{1}+p_{2}\left(p_{0}+p_{1}+u B_{3}\right) .
\end{aligned}
$$

Inasmuch as $A_{i}[1] \geqq A_{0}[1]$ for $i=1$ or 2 , and $A_{i}=B_{i}$ for $i=0,1,2$, we see that $B_{3}[1]$ must be distinct from $B_{k}[1]$ for some $k=0,1,2$, whence, by Lemma 1.3,

$$
u B_{3} \leqq B_{0} B_{1} B_{2} B_{3} \leqq B_{k} B_{3}=b_{i}+b_{j},
$$

and $u B_{3} \leqq p_{k}$ for this $k$. Thus

$$
\begin{aligned}
& u+a_{1}+p_{2}\left(p_{0}+p_{1}+u B_{3}\right)=u+a_{1}+p_{2}\left(p_{0}+p_{1}\right), \\
& a_{0} \leqq a_{1}+a_{0}\left(a_{1}+a_{2}\right)+p_{2}\left(p_{0}+p_{1}\right),
\end{aligned}
$$

and 


$$
a_{0}=a_{0}\left(a_{1}+a_{2}\right)+a_{0}\left(a_{1}+p_{2}\left(p_{0}+p_{1}\right)\right) .
$$

Since $a_{0}$ is a cycle, we conclude that $a_{0} \leqq a_{1}+a_{2}$ or $a_{0} \leqq a_{1}+p_{2}\left(p_{0}+p_{1}\right)$. However, inasmuch as $a_{0} \leqq a_{1}+a_{2}$ implies that $A_{1}, A_{2} \leqq A_{0}$, contradicting the assumption that $A_{i}[1] \not L A_{0}[1]$ for $i=1$ or 2 , we have the desired conclusion.

Definition. A lattice $L$ is said to be Arguesian if, for any six elements $a_{0}, a_{1}, a_{2}, b_{0}, b_{1}, b_{2}$ of $L$,

$$
\left(a_{0}+b_{0}\right)\left(a_{1}+b_{1}\right)\left(a_{2}+b_{2}\right) \leqq a_{0}\left(a_{1}+p_{2}\left(p_{0}+p_{1}\right)\right)+b_{0}\left(b_{1}+p_{2}\left(p_{0}+p_{1}\right)\right)
$$

where for $i, j, k$ distinct in $\{0,1,2\}, p_{i}=\left(a_{j}+a_{k}\right)\left(b_{j}+b_{k}\right)$.

Notation. We will denote the left and right hand sides of the inequality (11) by $l$ and $r$ respectively. For $i, j$ and $k$ distinct in $\{0,1,2\}$, we will write $g_{i}=\left(a_{j}+b_{j}\right)\left(a_{k}+b_{k}\right)$. If there is, in the same situation another ordered sextuplet $a_{0}^{\prime}, a_{1}^{\prime}, a_{2}^{\prime}, b_{0}^{\prime}, b_{1}^{\prime}, b_{2}^{\prime}$ of elements from the lattice $L$, we will denote the polynomials formed from them as above by $l^{\prime}, r^{\prime}, p_{i}^{\prime}$, and $g_{i}^{\prime}$.

THEOREM 1.6. A primary lattice $L$ of geometric dimension at least 4 is Arguesian.

Proof. Inasmuch as the formula (11) holds trivially for a sextuplet $a_{0}, a_{1}, a_{2}, b_{0}, b_{1}, b_{2}$ when one of the elements is an atom and the rest are zero, we can proceed by induction on $\sum_{0}^{2}\left(d\left[a_{i}\right]+d\left[b_{i}\right]\right)$. Letting $a_{0}^{\prime}=a_{0}\left(b_{0}+g_{0}\right)$, and $b_{0}^{\prime}=b_{0}\left(a_{0}+g_{0}\right)$, if $a_{0}^{\prime}<a_{0}$ or $b_{0}^{\prime}<b_{0}$, we can apply the inductive hypothesis to conclude that $l=l^{\prime} \leqq r^{\prime} \leqq r$. Thus, we might as well assume that $a_{0}^{\prime}=a_{0}$ and $b_{0}^{\prime}=b_{0}$, or, equivalently, that $a_{0}+g_{0}=b_{0}+g_{0}$. Similarly, we can assume that $a_{i}+g_{i}=b_{i}+g_{i}$, for $i=1,2$. Next suppose that $a_{0}$ is not a cycle. Then $a_{0}=a_{0}^{\prime}+a_{0}^{\prime \prime}$ where $a_{0}^{\prime}<a_{0}$ and $a_{0}^{\prime \prime}<a_{0}$, and, by the inductive hypothesis applied to $\left(a_{0}^{\prime}, a_{1}, a_{2}, b_{0}, b_{1}, b_{2}\right)$ and $\left(a_{0}^{\prime \prime}, a_{1}, a_{2}, b_{0}, b_{1}, b_{2}\right)$, we infer that $l^{\prime} \leqq r^{\prime} \leqq r$, and $l^{\prime \prime} \leqq r^{\prime \prime} \leqq r$. Inasmuch as $l^{\prime}+l^{\prime \prime}=l$, we conclude that $l \leqq r$. Thus we can assume that the elements $a_{0}, a_{1}, a_{2}, b_{0}, b_{1}, b_{2}$ are cycles. Finally, let $c$ be any cycle contained in $l$ and let $a_{i}^{\prime}=a_{i}\left(b_{i}+c\right)$ and $b_{i}^{\prime}=b_{i}\left(a_{i}+c\right)$ for $i=0,1,2$. It is easily shown that $a_{i}^{\prime}+b_{i}^{\prime}=a_{i}^{\prime}+c=$ $b_{i}^{\prime}+c$, so that, since $L$ is of geometric dimension at least 4 , we can apply Theorem 1.5 to conclude that $a_{0}^{\prime} \leqq a_{1}^{\prime}+p_{2}^{\prime}\left(p_{0}^{\prime}+p_{1}^{\prime}\right)$, and $b_{0}^{\prime} \leqq b_{1}^{\prime}+p_{2}^{\prime}\left(p_{0}^{\prime}+p_{1}^{\prime}\right)$. Thus $r \geqq r^{\prime}=a_{0}^{\prime}+b_{0}^{\prime} \geqq c$, and we have that $r$ contains every cycle that is contained in $l$. Inasmuch as $l$ is the sum of the cycles it contains, it follows that $r \geqq l$, as was to be shown.

2. In this section we exhibit an Arguesian primary lattice of 
geometric dimension 2 which cannot be represented as the lattice of submodules of a finitely generated module over a completely primary uniserial ring, thus showing that the assumption that $L$ be Arguesian or of geometric dimension at least 4 is necessary for the representation theorem.

Notation. The submodule lattice of a module $M$ will be denoted by $L(M)$. If $M$ consists of $n$-tuples from the ring $R$, we will denote by $\left[r_{1}, r_{2}, \cdots, r_{n}\right]$, the member of $L(M)$ spanned by the element $\left(r_{1}, r_{2}, \cdots, r_{n}\right)$.

We will consider throughout this section a fixed field $K$ and a one-to-one map $\varphi$ of $K$ onto itself such that $\varphi$ is not an automorphism but has the property: $\varphi(0)=0, \varphi(1)=1$. We also fix the vector space $V$ of 5 -tuples from $K$ and denote by $u_{i}$, the element of $V$ with 1 in the $i$ th place and 0 elsewhere. Then, letting $Q=\left[u_{1}\right]+\left[u_{2}\right]$, and $P=Q+\left[u_{3}\right]$ in $L(V)$, we define a one-to-one map of the elements of $L(V)$ covered by $Q$ onto the elements of $L(V)$ covering $P$ by

$$
\begin{aligned}
& F([r, 1,0,0,0])=P+[0,0,0, \varphi(r), 1], \\
& F\left(\left[u_{1}\right]\right)=P+\left[u_{4}\right] .
\end{aligned}
$$

Finally, we define the following subset of $L(V)$ :

$$
L_{\varphi}=[0, P] \cup[Q, V] \cup \bigcup_{X<Q}[X, F(X)] .
$$

We will refer to the intervals $[0, P],[Q, V]$ and $[X, F(X)]$ for $X \ll Q$ as the intervals used to define $L_{\varphi}$.

Observe that $[0, P]$ and $[Q, V]$ are subspace lattices of 3 -dimensional vector spaces over $K$ and hence can be viewed as subspace lattices of projective geometries $S_{1}$ and $S_{2}$ respectively. In this way $F$ is a one-to-one map of the points on the line $Q$ in $S_{1}$ to the lines containing the point $P$ of $S_{2}$. After showing that $L_{\varphi}$ is an Arguesian primary lattice of geometric dimension 2, we will prove that if $L_{\varphi}$ were representable as the submodule lattice of a finite dimensional module over a completely primary uniserial ring, then the function $F$ would have properties that imply that the map $\rho$ used to define it is an automorphism, thus contradicting our assumption on $\varphi$.

Theorem 2.1. The set $L_{\varphi}$ is a sublattice of $L(V)$ which is Arguesian and primary and is such that its identity element $V$ can be written as the sum of cycles:

$$
V=\left(\left[u_{1}\right]+\left(u_{4}\right]\right)+\left(\left[u_{2}\right]+\left[u_{5}\right]\right)+\left[u_{3}\right] .
$$

Proof. If the elements $x$ and $y$ of $L_{\varphi}$ are in a common interval 
used to define it, then clearly their sum and product are in $L_{\varphi}$. On the other hand, if $x \in[A, B]$, and $y \in[C, D]$, then $x+y \in[A+C, B+D]$, and $x y \in[A C, B D]$. Inasmuch as the set of intervals used to define $L_{\varphi}$ is closed under the operations

$$
\begin{aligned}
& {[A, B] \oplus[C, D]=[A+C, B+D],} \\
& {[A, B] \bullet[C, D]=[A C, B D],}
\end{aligned}
$$

$L_{0}$ is a sublattice of $L(V)$. Showing tnat $\mathrm{L}_{\varphi}$ is semi-primary clearly reduces, by symmetry, to showing that every element $A$ of $L_{0}$ is the sum of cycles of $L_{\varphi}$. Inasmuch as an element in a complemented lattice is the sum of atoms, a proof by induction on $d[A]$ clearly reduces to showing that if $A$ is an atom of one of the intervals used to define $L_{\varphi}$ and in no other such interval, then $A$ is a cycle. This is clearly true of atoms of $[0, P]$ and $[X, F(X)]$ for $X \ll Q$, so we can assume that $A$ is an atom of $[Q, V]$. But then either $A=P \in[0, P]$ or $A+P \gg P$, whence $A+P=F(X)$ for some $X \ll Q$, and $A \in[X, F(X)]$. In either event we have a contradiction, and we conclude that $L_{\varphi}$ is semi-primary.

To see that $L_{\varphi}$ is primary, note first that this reduces to showing that an interval of length 2 cannot have exactly two atoms, and note further that an interval in the lattice of subspaces of a vector space is itself the subspace lattice of a vector space and can therefore be shown to have at least three atoms. Thus, proving that $L_{\varphi}$ is primary reduces to proving that every interval $[A, B]$ of length 2 with distinct atoms $X_{1}$ and $X_{2}$ is contained in one of the intervals used to define $L_{\varphi}$. We might as well assume then that the elements $X_{1}$ and $X_{2}$ are in distinct intervals $\left[C_{1}, D_{1}\right]$ and $\left[C_{2}, D_{2}\right]$ used to define $L_{0}$ for otherwise we would be through. By symmetry this reduces to three cases: (i) $C_{1}=0, C_{2}=Q$, (ii) $C_{2} \ll C_{1}, D_{2} \ll D_{1}$, (iii) $C_{1}, C_{2} \ll Q$. However, we can immediately dismiss the first case because, since $X_{1}$ and $X_{2}$ are of the same dimension, we have that $X_{1}=P \in[Q, V]$ or $X_{2}=Q \in[0, P]$. Since $X_{2} \ll X_{1}+X_{2}$ we infer that $X_{2}=\left(X_{1}+X_{2}\right) D_{2}$ or $X_{1}+X_{2} \leqq X_{2}$, whence $X_{2} \geqq\left(C_{1}+C_{2}\right) D_{2}=C_{2}+C_{1} D_{2}$ or $X_{1} \leqq D_{2}$. In case (ii) $C_{1} \leqq D_{2}$, so that $X_{2} \geqq C_{1}$ or $X_{1} \leqq D_{2}$ and we are through while, in case (iii) $C_{1}+C_{2}=Q$ and $D_{1} D_{2}=P$ so that $X_{2} \in[Q, V]$ or $X_{1} \in[0, P]$ which is case (ii). Thus $L_{\varphi}$ is primary.

That $L_{0}$ is Arguesian follows from the fact that it is a sublattice of $L(V)$ which, by [2; Th. 2.14], is Arguesian and the observation that the condition defining Arguesian lattices can be written as an identity.

Finally, note that, since $\left[u_{1}\right]+\left[u_{4}\right]$ is not contained in $P$, it cannot be the sum of atoms of $L_{c}$. But since $\left[u_{1}\right]+\left[u_{4}\right]$ is of dimension 2, this means that it must be a 2 -cycle in $L_{\varphi}$. Similarly $\left[u_{2}\right]+\left[u_{5}\right]$ is 
a 2-cycle in $L_{\varphi}$. Inasmuch as the atoms of $L_{\varphi}$ contained in the cycles $\left[u_{1}\right]+\left[u_{4}\right],\left[u_{2}\right]+\left[u_{5}\right]$ and $\left[u_{3}\right]$ are $\left[u_{1}\right],\left[u_{2}\right]$ and $\left[u_{3}\right]$ respectively we conclude from 4.14 of PAL that these cycles are independent, whence, by a dimension argument.

$$
V=\left(\left[u_{1}\right]+\left[u_{4}\right]\right)+\left(\left[u_{2}\right]+\left[u_{5}\right]\right)+\left[u_{3}\right] .
$$

Lemma 2.2. Given an element $X$ of $L_{\varphi}$ that is covered by $Q$, there is a 2-cycle $X^{\prime}$ of $L_{\varphi}$ covering $X$. Further, for any 2-cycle $X^{\prime \prime}$ covering $X, F(X)=X^{\prime \prime}+P$.

Proof. Inasmuch an $[X, F(X)]$ is complemented, $F(X)$ is the sum of elements covering $X$. If none of these elements is a 2-cycle of $L_{\varphi}$, then each must be the sum of atoms, and $F(X)$ is the sum of atoms. This contradicts the fact that $F(X)$ covers $P$, and every atom of $L_{\varphi}$ is contained in $P$. Thus there is a 2-cycle $X^{\prime}$ covering $X$. Now, if $X^{\prime \prime}$ is any 2-cycle covering $X$, since $[0, P]$ is complemented we infer that $X^{\prime \prime} \$ P$. Thus, either $X^{\prime \prime} \leqq F(X)$, and $X^{\prime \prime}+P=F(X)$, or $X^{\prime \prime} \not \equiv F(X)$, and $X^{\prime \prime}+F(X)=V$. However, in the latter case $V$ is the sum of elements covering $X$, whence $[X, V]$ is complemented, and $X$ is the product of elements covered by $V$. Inasmuch as the only dual atoms of $L_{\varphi}$ are in $[Q, V]$, this implies that $X \geqq Q$, a contradiction.

Lemma 2.3. If $L_{\varphi}$ is representable as the submodule lattice of a finitely generated module over a completely primary uniserial ring, then there is an isomorphism \& of $[0, P]$ onto $[Q, V]$ such that

(a) $\psi\left[u_{1}\right]=\left[u_{4}\right]+Q, \psi\left[u_{2}\right]=\left[u_{5}\right]+Q, \psi\left[u_{3}\right]=\left[u_{3}\right]+Q$,

(b) $F(X)=\psi(X)+P$ for every $X \ll Q$.

Proof. Suppose $\lambda^{\prime}$ is an isomorphism of $L_{\varphi}$ onto the submodule lattice of a module $M^{\prime}$ over a completely primary uniserial ring $R$. For definiteness we will take $M^{\prime}$ to be a left $R$-module, although it will be apparent from the proof that there is no loss of generality in this assumption. Furthermore, since $L_{\varphi}$ if of rank 2, we can view $M^{\prime}$ as a module over $R / J^{2}$ or, equivalently, assume that $J^{2}=(0)$. Then, since

$$
M^{\prime}=\lambda^{\prime}\left(\left[u_{1}\right]+\left[u_{4}\right]\right) \oplus \lambda^{\prime}\left(\left[u_{2}\right]+\left[u_{5}\right]\right) \oplus \lambda^{\prime}\left(\left[u_{3}\right]\right),
$$

and $R$ is completely primary and uniserial, there is an isomorphism $\lambda$ of $L_{\varphi}$ onto $L(M)$, where $M=R \times R \times R / J$, such that

$$
\lambda\left(\left[u_{1}\right]+\left[u_{4}\right]\right)=\left[m_{1}\right], \lambda\left(\left[u_{2}\right]+\left[u_{5}\right]\right)=\left[m_{2}\right], \lambda\left(\left[u_{3}\right]\right)=\left[m_{3}\right],
$$

where, by $m_{i}$ we mean the element of $M$ with 1 in the $i$ th place 
and 0 elsewhere. If we fix a generator $p$ of $J$, it is clear that [pm $]$ and $\left[p m_{2}\right]$ are the unique elements of $L(M)$ covered by $\left[m_{1}\right]$ and $\left[m_{2}\right]$ respectively. Thus, since $\left[u_{1}\right]$ and $\left[u_{2}\right]$ are the unique elements of $L_{\varphi}$ covered by $\left[u_{1}\right]+\left[u_{4}\right]$ and $\left[u_{2}\right]+\left[u_{5}\right]$, it follows that

$$
\lambda\left[u_{1}\right]=\left[p m_{1}\right], \lambda\left[u_{1}\right]=\left[p m_{2}\right]
$$

and if, $Q^{\prime}=\lambda(Q)$ and $P^{\prime}=\lambda(P)$, then

$$
Q^{\prime}=\left[p m_{1}\right]+\left[p m_{2}\right], P^{\prime}=Q^{\prime}+\left[m_{3}\right] \text {. }
$$

Moreover,

$$
\lambda\left(\left[u_{4}\right]+Q\right)=\lambda\left(\left[u_{1}\right]+\left[u_{4}\right]+Q\right)=\left[m_{1}\right]+Q, \lambda\left(\left[u_{5}\right]+Q\right)=\left[m_{2}\right]+Q^{\prime} .
$$

Inasmuch as $Q^{\prime}=\left[p m_{1}\right]+\left[p m_{2}\right]$, the elements of $L(M)$ covered by $Q^{\prime}$ are of the form $\left[p r_{1}, p r_{2}, 0\right]$ for $r_{1}$ and $r_{2}$ in $R$ such that $r_{1}$ or $r_{2}$ is not in $J$. Clearly, $\left[r_{1}, r_{2}, 0\right]$ is a 2-cycle covering such an element, so that, if we define $F^{\prime}=\lambda F \lambda^{-1}$, according to 2.2 ,

$$
F^{\prime}\left[p r_{1}, p r_{2}, 0\right]=\left[r_{1}, r_{2}, 0\right]+P^{\prime},
$$

for $r_{1}, r_{2} \in R$ with $r_{1}$ or $r_{2} \notin J$.

Since $J P^{\prime}=0$ and $J M \cong Q^{\prime}, P^{\prime}$ and $M / Q^{\prime}$ are modules over the division ring $R / J$ with bases $\left\{p m_{1}, p m_{2}, m_{3}\right\}$ and $\left\{m_{1}+Q^{\prime}, m_{2}+Q^{\prime} m_{3}+Q^{\prime}\right\}$ respectively. Thus the correspondence

$$
p m_{1} \longrightarrow m_{1}+Q^{\prime}, p m_{2} \longrightarrow m_{2}+Q^{\prime}, m_{3} \longrightarrow m_{3}+Q^{\prime}
$$

defines a lattice isomorphism $\psi^{\prime}:\left[0, P^{\prime}\right] \cong\left[Q^{\prime}, M\right]$ which has the properties

$$
\begin{aligned}
& \psi^{\prime}\left[p m_{1}\right]=\left[m_{1}\right]+Q^{\prime}, \psi^{\prime}\left[p m_{2}\right]=\left[m_{2}\right]+Q^{\prime}, \psi^{\prime}\left[m_{3}\right]=\left[m_{3}\right]+Q^{\prime} \\
& \psi^{\prime}\left[p r_{1}, p r_{2}, 0\right]=\left[r_{1}, r_{2}, 0\right]+Q^{\prime},
\end{aligned}
$$

so that, by (1), for every $X \ll Q^{\prime}$,

$$
F^{\prime}\left(X^{\prime}\right)=\psi^{\prime}\left(X^{\prime}\right)+P^{\prime} \text {. }
$$

Therefore, defining $\psi=\lambda^{-1} \psi^{\prime} \lambda$, we have

$$
\psi\left[u_{1}\right]=\left[u_{4}\right]+Q, \psi\left[u_{2}\right]=\left[u_{5}\right]+Q, \psi\left[u_{3}\right]=\left[u_{3}\right]+Q .
$$

Taking $X^{\prime}$ to be $\lambda(X)$ in (2) for $X \ll Q$, we also have $F^{\prime} \lambda(X)=$ $\psi^{\prime} \lambda(X)+P^{\prime}$ so that, applying $\lambda^{-1}$ to both sides, we conclude that $F(X)=\psi(X)+P$, as was to be shown.

THEOREM 2.4. The lattice $L_{\varphi}$ cannot be represented as a submodule lattice of a finitely generated module over a completely primary uniserial ring. 
Proof. Denoting by $V_{3}$, the vector space of triples from $K$, and by $w_{i}$ the element of $V_{3}$ with 1 in the $i$ th place and zero elsewhere, it is clear that the correspondences $\left\{w_{i} \rightarrow u_{i}\right\}_{i=1}^{3}$ and

$$
\left\{w_{1} \longrightarrow u_{4}+Q, w_{2} \longrightarrow u_{5}+Q, w_{3} \longrightarrow u_{3}+Q\right\}
$$

give lattice isomorphisms $\sigma$ and $\tau$ of $L\left(V_{3}\right)$ onto $[0, P]$ and $[Q, V]$ respectively, so that the map $\theta=\tau^{-1} \psi \sigma$ is an automorphism of $L\left(V_{3}\right)$ with $\theta\left[w_{i}\right]=\left[w_{i}\right]$ for $i=1,2,3$, and

$$
F \sigma[r, 1,0]=\tau[\varphi(r), 1,0]+\tau\left[w_{3}\right] .
$$

However, in view of 2.3 ,

$$
F \sigma[r, 1,0]=\psi \sigma[r, 1,0]+\psi \sigma\left[w_{3}\right],
$$

whence

$$
\psi \sigma[r, 1,0]+\psi \sigma\left[w_{3}\right]=\tau[\varphi(r), 1,0]+\tau\left[w_{3}\right],
$$

and, applying $\tau^{-1}$ to both sides of this equation, we conclude that

$$
\theta[r, 1,0]+\theta\left[w_{3}\right]=[\varphi(r), 1,0]+\left[w_{3}\right],
$$

and $\theta[r, 1,0]=[\varphi(r), 1,0]$. According to the fundamental theorem of projective geometry, $\theta$ is induced by a semilinear transformation $\left(T, \varphi^{\prime}\right)$ where $T$ is an automorphism of the additive group of $V_{3}$ and $\varphi^{\prime}$ is an automorphism of $K$. Since $\theta\left[w_{i}\right]=\left[w_{i}\right]$ for $i=1,2,3$, there are nonzero elements $s_{1}, s_{2}, s_{3}$ in $K$ such that $T\left(w_{i}\right)=s_{i} w_{i}$ for $i=1,2,3$. On the other hand,

$$
\begin{aligned}
{[\varphi(r), 1,0] } & =\theta[r, 1,0]=[T(r, 1,0)]=\left[\varphi^{\prime}(r) T\left(w_{1}\right)+T\left(w_{2}\right)\right] \\
& =\left[\left(\varphi^{\prime}(r) s_{1}, s_{2}, 0\right],\right.
\end{aligned}
$$

so that $\varphi^{\prime}(r) s_{1}=\varphi(r) s_{2}$. However, since $\varphi^{\prime}(1)=1=\varphi(1)$, we see that $s_{1}=s_{2}$, whence $\varphi^{\prime}(r)=\varphi(r)$. Thus $\varphi$ must be an automorphism, contradicting the original assumption on $\varphi$, and the theorem is established.

\section{BIBLIOGRAPHY}

1. E. Inaba, On primary lattices, Journal of the Faculty of Science, Hokkaido University 11 (1948), 39-107.

2. B. Jónsson, Modular lattices and Desargues' throrem, Math. Scand. 2 (1953), 205-314.

3. B. Jónsson and G. Monk, Representations of primary Arguesian lattices (to appear.)

Received June 24, 1968. Research supported by National Science Foundation grant GP-6545. 


\section{PACIFIC JOURNAL OF MATHEMATICS}

\section{EDITORS}

H. ROYDEN

Stanford University

Stanford, California

Richard Pierce

University of Washington

Seattle, Washington 98105
J. DugundJI

Department of Mathematics

University of Southern California

Los Angeles, California 90007

BASIL GORDON

University of California

Los Angeles, California 90024

\section{ASSOCIATE EDITORS}

E. F. BECKENBACH

B. H. NeUmanN

F. WOLF

K. YOSHIDA

\section{SUPPORTING INSTITUTIONS}

UNIVERSITY OF BRITISH COLUMBIA

CALIFORNIA INSTITUTE OF TECHNOLOGY

UNIVERSITY OF CALIFORNIA

MONTANA STATE UNIVERSITY

UNIVERSITY OF NEVADA

NEW MEXICO STATE UNIVERSITY

OREGON STATE UNIVERSITY

UNIVERSITY OF OREGON

OSAKA UNIVERSITY

UNIVERSITY OF SOUTHERN CALIFORNIA
STANFORD UNIVERSITY

UNIVERSITY OF TOKYO

UNIVERSITY OF UTAH

WASHINGTON STATE UNIVERSITY

UNIVERSITY OF WASHINGTON

$* * \quad * \quad *$
AMERICAN MATHEMATICAL SOCIETY
CHEVRON RESEARCH CORPORATION
TRW SYSTEMS
NAVAL WEAPONS CENTER




\section{Pacific Journal of Mathematics}

Vol. 30, No. $1 \quad$ September, 1969

William Wells Adams, Simultaneous diophantine approximations and cubic irrationals ..................................... 1

Heinz Bauer and Herbert Stanley Bear, Jr., The part metric in convex

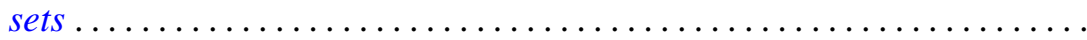

L. Carlitz, A note on exponential sums ...................... 35

Vasily Cateforis, On regular self-injective rings ................. 39

Franz Harpain and Maurice Sion, A representation theorem for measures on infinite dimensional spaces ......................... 47

Richard Earl Hodel, Sum theorems for topological spaces .............. 59

Carl Groos Jockusch, Jr. and Thomas Graham McLaughlin, Countable

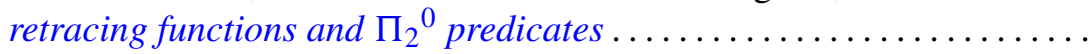

Bjarni Jónsson and George Stephen Monk, Representations of primary

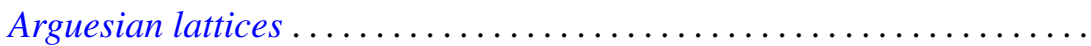

Virginia E. Walsh Knight, A continuous partial order for Peano continua...................................... 141

Kjeld Laursen, Ideal structure in generalized group algebras ........... 155

G. S. Monk, Desargues' law and the representation of primary lattices . . . 175

Hussain Sayid Nur, Singular perturbation of linear partial differential equation with constant coefficients ..........................

Richard Paul Osborne and J. L. Stern, Covering manifolds with cells ... 201

Keith Lowell Phillips and Mitchell Herbert Taibleson, Singular integrals in several variables over a local field...

James Reaves Smith, Local domains with topologically $T$-nilpotent radical....

Donald Platte Squier, Elliptic differential equations with discontinuous coefficients .................................

Tae-il Suh, Algebras formed by the Zorn vector matrix...

Earl J. Taft, Ideals in admissible algebras . .................... 259

Jun Tomiyama, On the tensor products of von Neumann algebras........ 263

David Bertram Wales, Uniqueness of the graph of a rank three group ..... 271

Charles Robert Warner and Robert James Whitley, A characterization of regular maximal ideals ......................... 